In "The Structure of Microtubules" Amos presents a detailed review of results from X-ray diffraction, electron microscopy and image reconstruction. Much of the discussion is technical in nature, but for those interested in this area the article is especially timely, since the recently obtained $2 \mathrm{~nm}$ resolution is close to the limit of specimens and techniques currently available. "The Biochemistry of Tubulin" by Ludueña should be useful as a summary of basic information and an introduction to the literature. The biochemistry of tubulin and nucleotides, an area of increasing importance in tubulin research, is expanded in a separate chapter by Jacobs.
In vitro assembly of microtubules is covered by Scheele and Borisy in a single chapter, which is devoted largely to work from their own laboratory. Their treatment of the thermodynamics and kinetics of assembly is quite useful but their lengthy discussion of tubulin rings seems out of proportion to the importance of this subject as it is currently understood. A discussion of the assembly of purified (MAP-free) tubulin and the effect of solution conditions and ligands would have been much more valuable. This important area, which could easily have merited a full chapter, is virtually ignored in the book.

This volume should be useful to people in the microtubule field, providing them with an opportunity to review the advances of the last 15 years in areas related to but outside their immediate interests. For the person unfamiliar with microtubule research it would be difficult for any publication to provide easy access to quick answers, largely because the field is now so complex. For the student who wants to consider the subject in some depth, however, this book should be useful as a fairly comprehensive summary of results and an introduction to the literature up to 1978.

H.P. Erickson is Associate Professor in the Department of Anatomy, Duke University, Durham, North Carolina.

\section{Solid-state spectroscopy}

\section{Derek J. Fabian}

Electron Spectroscopy of Crystals. By V.V. Nemoshkslenko and V.G. Aleshin (translated from Russian by Irina Curelaru). Pp.360. (Plenum: New York and London, 1979.) £31.19.

THE major part of this monograph comprises an extensive review of published photoelectron emission data covering a range of band structure effects in solids and solid surfaces. The book includes abundant introductory theory, of assistance to the reader both in understanding the electron transition processes involved in photoemission and Auger spectroscopies and in following the interpretations offered for many of the experimental observations.

To some extent the word 'crystals' in the title should be taken to mean 'solids', for the review covers ordered and disordered metals and alloys as well as including intermetallic and non-metallic amorphous materials. On the other hand the emphasis is largely on crystalline solids, with separate chapters devoted to sphaleritelattice compounds, alkali and alkalineearth halides, and transition-metal compounds.

The text commences with a helpful description of commercially available electron spectrometers, which unfortunately was already dated at the time of the original Russian version of the book in 1976. An excellent chapter on background theory - misleadingly headed "Physical principles of electron spectroscopy" provides, for the newcomer to the field, a definitive introduction to theoretical principles of photoelectron ionization and emission, together with a descriptive outline of computative methods used for calculating energy bands in solids. The chapter on metals and alloys also contains valuable descriptive theory, including the coherent potential method in the study of disordered systems. Further chapters deal with characterization of surfaces, including some novel aspects of quantitative analysis using angularly resolved photoemission, and with multiplet and satellite structure in photoelectron core-level spectra.

A welcome theme, throughout the review of experimental data, is the comparison of band-structure information gained from X-ray photoelectron spectra with that obtained from X-ray emission and absorption studies. Photoelectron and $X$-ray emission spectroscopies when combined can provide a powerful tool for probing occupied energy bands in solids. This is especially well-illustrated in the chapter dealing with core-electron and valence-band energy levels in transitionmetal compounds. One topic, though, not covered adequately in the book is the determination of partial densities of electron states with various symmetries from angular distributions of emitted photoelectrons.

The monograph contains an extensive bibliography, which is particularly

\section{Marking tumours}

\section{A. Munro Neville}

Cancer Markers. Edited by S. Sell. Pp.541. (Humana Press: Clifton, New Jersey, 1980.) $\$ 49.50$.

THE past decade has witnessed a great deal of progress in the search for biochemical markers of neoplasia. In view of their potential biological, pathological and clinical importance, it is most welcome to have a book where most of the relevant data can be found. All the chapters are informative, easily read and in the main remarkably up to date.

Among the wide range of topics discussed are aspects of alphafetoprotein (AFP), the carcinoembryonic antigen comprehensive in its coverage of experimental work published in the USSR; however, its usefulness would have been enhanced by the inclusion of an author index. The book has a subject index and this too could have been improved had more detail been included. These are minor criticisms that do not detract significantly from the scientific quality of the text, which also reads lucidly and smoothly with little evidence that the original was not in English. The book can be firmly recommended both as an introductory work to students and as a valuable review to established researchers in the field. The authors set out to provide a comprehensive survey of experimental energy-band data based on photoelectron spectroscopies, drawing on their own noted research at the Ukrainian Academy of Sciences in Kiev, and to fill a gap in published monographs and reviews on electron spectroscopy applied to solid-state materials. To a welcome extent they have succeeded.

Derek J. Fabian is Reader in Metallurgy and Materials Physics at the University of Strathclyde, Glasgow, UK.

(CEA), lymphocyte markers and pregnancy proteins, all of which are discussed from a fundamental point of view as well as in clinical terms.

Most of the other currently recognized human solid tumour-associated antigens are gathered together in one chapter thereby giving a good reference source, albeit without critical comment. The chapter on teratomas in particular is a fascinating and thought-provoking account, and the section dealing with lectins and their use as probes to dissect cell surfaces is most valuable.

This is a book of great interest to all concerned with the field of tumour markers, is timely in its publication and is highly recommended.

A. Munro Neville is a Professor at the Ludwig Institute for Cancer Research, Royal Marsden Hospital, Sutton, UK. 